\title{
Device based QoS Approach for Multimedia Proxy Handoff in Mobile Networks
}

\author{
M. Naveen Kumar \\ Assistant professor \\ Department of Computer Science and Engineering \\ Saveetha Engineering College
}

\author{
R. Suresh \\ Assistant professor \\ Department of Information Technology \\ SAMS College of Engineering and Technology
}

\begin{abstract}
Layer streaming has received extensive attention due to the highly heterogeneous access to the Internet. In the three-tier networking architecture, proxies can improve the quality of service of clients. The system performance can further be improved via co-operation of the proxies. Here, user mobility is taken into consideration, i.e., mobile clients can switch to a proxy dynamically. Proxy Handoff is defined to have applications executed smoothly when mobile clients move in the server-proxy-client architecture. First, proxy handoff employs application-layer anycast to select one of the candidate proxies as the next proxy based on 1) the network condition between the mobile client and each candidate proxy and 2) the load balance among the candidate proxies.
\end{abstract}

\section{Keywords}

Handoff protocol, proxy, proxy handoff algorithm, proxy selection algorithm

\section{INTRODUCTION}

Handoff is the process of transferring an ongoing call or data session from one base station to another base station without loss or disruption of service. Vertical handoffs can be classified into Upward and Downward Handoffs, Hard and Soft handoffs, Imperative and Alternative handoffs [3]. Vertical handoffs can further be classified based on who controls the handoff decision. If mobile node controls the handoff decision, it is termed as Mobile controlled handoff (MCHO). In Network controlled Handoff (NCHO) networks control the handoff decision [3]. There are two types of handoffs that have been widely discussed for mobile networks: layer 2 (L2) handoff and layer3 (L3) handoff. L2 handoff [1], which is normally known as access point (AP) handoff or base station (BS) handoff, means that a mobile host $(\mathrm{MH})$ goes beyond the range of the associated AP and must associate with a new AP in order to keep accessing the Internet. Common method to perform L2 handoff is based on signal strength. Once a $\mathrm{MH}$ detects that signal strength of the current AP is too weak, it usually associates with another AP which has stronger signal strength. L3 handoff, which is normally known as IP handoff, means that a $\mathrm{MH}$ goes from one subnet to another subnet and these two subnets belong to different IP domains in network.

Mobile IP is the most popular method for solving L3 handoff is Mobile IP [4]. Mobile IP uses two IP addresses, i.e., home address and care-of-address. The home address of a $\mathrm{MH}$ is always the same no matter where it is located. However, the MH should get a new care-of-address when it attaches to a new subnet. Once the care-of-address is changed, the MH must inform its home agent about the new care-ofaddress in order to make the mapping between its home address and its care-of-address correct.
Here L2 handoff keeps the accessibility of a MH and L3 handoff remains the IP connectivity of a MH, neither L2 handoff nor L3 handoff considers and supports this kind of handoff, i.e., switching to another proxy dynamically when a MH keeps moving all the time. Thus, the aforementioned handoff is defined as one kind of layer7 (L7) handoff in this

paper. To overcome the drawbacks of above handoff, a new mechanism, called Application-layer Proxy Handoff (APH), is proposed to deal with switching from one proxy to another. To propose APH is devised in the IPv6 wireless/ mobile network environment. Two features that APH adopts and utilizes are 1) application-layer anycast 2) IPv6 multicast.

Application-layer anycast is adopted to resolve the proxy selection concern. When a MH is getting a worse service from the current proxy, it may have more than one candidate proxy that can be qualified as the next proxy. IPv6 multicast is utilized to transmit the requested multimedia data from the server to the corresponding proxies When a MH moves to a new subnet and has selected the next proxy, APH makes the next proxy join the corresponding multicast group and has the original proxy forward the available cache to the next proxy for resuming the status of the original session in the next proxy. Application-layer Proxy Handoff (APH) is defined to have applications be executed smoothly when mobile clients move in the server-proxy-client architecture.

First, APH employs application-layer anycast to select one of the candidate proxies as the next proxy based on the network condition between the mobile client and each candidate proxy and the load balance among the candidate proxies. Second, APH utilizes IPv6 multicast to switch the session from the original proxy to the next proxy smoothly and to forward the available cache unsent in the original proxy to the next proxy for keeping the original session continuous. The newly added proxy continuously keep the original session.

The rest of the paper is organized as follows section I describes about the introduction for proxy handoff. Section II describes about the need for proxy handoff. Section III describes the protocols of handoff. Section IV describes about the anycast metrics for proxy selection. Section V describes about the proxy selection algorithm. Section VI describes about proxy handoff procedure. Section VII describes about Performance Analysis.

\section{INTRODUCTION TO L7 HANDOFF}

L7 handoff is called as Layer7 handoff. This can be divided into two categories, 1) handoff using the L7 method and 2) handoff behavior of applications. Handoff using the L7 
method [5] means that the method to resolve the IP connectivity of a $\mathrm{MH}$ is within the application layer, not the network layer. A popular handoff mechanism using the L7 method is SIP mobility [6]. Originally, Session Initiation Protocol (SIP) was designed to initiate, maintain, and terminate a session between SIP clients. When SIP is applied to mobile networks, it can also handle the IP connectivity problem. Once a $\mathrm{MH}$ connects to a new subnet and gets a new IP address, it should register its new IP address using the SIP REGISTER method and reinvest the correspondence node $(\mathrm{CN})$, which was originally communicating with the $\mathrm{MH}$, using the SIP INVITE method.

However, this kind of L7 handoff does not need to change the current IP stack and can support user mobility. Handoff behavior of applications means that applications should adjust their status according to user mobility. Proxies can play the role of handling the handoff behavior of applications [7]. For example, a $\mathrm{MH}$ that is getting a network service from the Internet moves from a wired network to a wireless network. Since these two networks are heterogeneous, the MH seems unable to get the service with the same quality.

If this $\mathrm{MH}$ gets the network service via a proxy, the proxy can help the service be adaptive to the network environment, i.e., changing the quality of the requested data dynamically. Thus, this kind of L7 handoff can improve the quality of service (Qos) of applications.

\section{HANDOFF PROTOCOLS}

This tells that When a MH keeps moving and browsing the Web, it needs to change the current Web proxy due to the performance concern. Kim et al. [8] proposed a handoff (HO) message protocol. When a MH moves to another region, the HO message protocol can enable handoffs between proxies. The handoff process consists of three steps 1) the proxy handoff initiation 2) distilled data synchronization between two proxies 3 ) the handoff processing between the proxy and the Web server.

First, the MH issues a handoff request message containing the list of files that must be received but are not yet received by this MH to the current proxy. Then, the current proxy may request the previous proxy to synchronize some of those files that are received and distilled by the previous proxy. Finally, the current proxy requests the files that have not been received by the previous proxy from the Web server. Other important handoff protocol was proposed by Gu and Helal [9] .They proposed an Extended Internet Caching Protocol (X-ICP). XICP is based on Mobile IP protocol and allows the newly attached proxy (foreign proxy) to fetch a copy from a home proxy. Thus, if the foreign proxy caches no data for the MH, it forwards the request to the home proxy. If the home proxy also caches no data, it forwards the request to the corresponding Web server. Content distribution from a single source (A. Dutta, J. Chennikara) follows the one-to-many model. Most broadcasting sources such as radio and TV networks follow this kind of model [10]. Multicasting streaming content to end users over the Internet may include both mobile and nonmobile clients over wired and wireless media.

A handover based traffic management algorithm has been proposed by (Nandha kumar et al, 2011) (\$ different reference styles have been used in different places) in which the traffic load has been considered and using this algorithm the service quality has been improved [2]. In addition, the traffic distribution has been used to maintain service quality [2].An optimal network and the QOS has been improved using vertical handoff algorithm [3] proposed by (Radhika, venugopal Reddy 2011). In addition, the handoff delay has been reduced.

When the MH leaves the foreign proxy, the foreign proxy should send back the complete object list of this $\mathrm{MH}$ to the home proxy. Hence, when the MH connects to another foreign proxy, the new foreign proxy can get the data from the home proxy. The difference between the above two methods is that the current proxy in the $\mathrm{HO}$ message protocol fetches some contents from the previous proxy but the one in X-ICP is from the home proxy.

\section{ANYCAST METRICS}

Anycast metrics are defined to select the appropriate node according to user requirements. In this paper, anycast metrics are defined to select the most suitable LMP as the next LMP. On the client's perspective, the most suitable LMP for streaming service means that an $\mathrm{MMH}$ can receive the requested stream from this LMP with less delay, less jitter, and less packet loss. Therefore, two metrics in the proposed proxy selection scheme are 1) workload and 2) round-trip time. Workload is considered to avoid that one LMP serves too many MMHs to result in the long system response time. Round-trip time is considered to measure the transmission condition between an MMH and an LMP. Any cast metrics Equation (1) is formulated based on two metrics.

Anycast metrics $=\mathrm{Min}\left(\mathrm{W} * \mathrm{LOAD}_{\mathrm{i}}+(1-\mathrm{W}) * \mathrm{RTT}_{\mathrm{i}}\right)-----(1)$

Where $\mathrm{i}$ is some LMP in an RPG, $\mathrm{W}(0<\mathrm{W}<1)$ is the weight value, LOADi denotes the workload of $i$, and RTTi denotes the round-trip time of $i$. The LMP with the minimum value of (1) is selected to be the next LMP. In (1), the weight value, i.e., $\mathrm{W}$, can be adjusted by the administrator in different network situations. If $\mathrm{W}$ is larger,i.e., LOADi with greater weight, it implies that the whole system wants to distribute the load of each LMP and to achieve load balance as soon as possible for keeping the whole system stable.

On the other hand, if $\mathrm{W}$ is smaller, i.e., RTTi with greater weight, it implies that the whole system is stable and can optimize the transmission rate of each MMH. Hence, W keeps the proxy selection scheme flexible in different network situations.

Since the network condition of each MMH is different from each other, each MMH selects its next LMP individually using (1). That is, when each MMH recognizes that it needs to select a new LMP, it would send an anycast request to the default ADN, i.e., the predefined site-local multicast address. All LMPs of the corresponding RPG respond with their metrics to each requesting MMH, respectively.

\section{PROXY HANDOFF ALGORITHM}

The proposed proxy selection algorithm is divided into two steps. The first step is to define the time point of executing the proxy selection algorithm. Algorithm 1 depicts two trigger time points. This indicates the time point at which an $\mathrm{MMH}$ is recommended to execute the proxy selection algorithm because it crosses the service area of the original LMP. APH assumes that each LMP has its service area. MMHs in the service area of an LMP can get better quality of service than that of the service area of the same LMP. 
A MMH is recommended to select a new LMP after crossing the service area of the original LMP. This indicates the point in time at which an MMH decides to execute the proxy selection algorithm because it detects its bad transmission quality. For example, MMH X selects LMP A as its serving LMP. However, the workload of LMP A is increasing as time goes on. LMP A may not provide the best quality of service for MMH X. Thus, an MMH may want to reselect an LMP even if in the no movement situation.

\section{ALGORITHM}

Step: 1 select the IP address (IP).

Step: 2 select the service area (SA)

Step: 3 If MMH crosses from the original service area go to proxy selection algorithm.

Step: 4 If $\mathrm{MMH}$ crosses from the original subnet go to proxy selection algorithm.

Step: 5 If MMH gets bad transmission from the original proxy go to proxy selection algorithm.

\section{PROXY SELECTION ALGORITHM}

Step: 1 Get the response time (T).

Step: 2 Select any LMP.

Step: 3 If T has maximum waiting time

Step: 4 MMH Sends anycast request to AND

Step: 5 Get load of LMP

Step: 6. Get response time of LMP.

Step: 7. Get weight value

Step: 8. Calculate the metric value.

Step: 9. if the metric value LMPA is Minimum.

Step: 10 . Then select LMP

\section{PROXY HANDOFF PROCEDURE}

This tells about the handoff procedure when the MMH moves out of the service area of the current LMP, L2 or L3 handoff should be executed at first. After the L2/L3 handoff, the MMH reconnects to the original LMP and still receives the original session. However, since the MMH crosses the service area of the current LMP, it is recommended to perform the proposed $\mathrm{PH}$ in this paper and then switch to the next LMP.

1. The MMH sends an anycast request to the predefined ADN for deriving the metric information.

2. When each LMP of the next RPG receives an any cast Request, it starts to calculate its workload.

3. Each LMP reports its workload to the MMH.

4. When the MMH receives all of the responses in the Countdown interval, it selects the LMP that has the minimum Value of (1) to be the next LMP. The MMH Registers to the next LMP and informs the next LMP to join the corresponding multicast group in order to receive media packets continuously.

5. The next LMP sends an ACK message back to the MM after joining the corresponding multicast group.

6. The MMH informs the original LMP that the MMH will stop receiving media packets from it.

7. The original LMP checks its cache and identifies which packets are unsent.

8. The original LMP starts to perform the buffer forwarding process.

a. The original LMP replies to the MMH and stops sending packets to it. b. The original LMP leaves the corresponding multicast group.

c. The original LMP sends a Hello message to the next LMP in order to prepare to forward the unsent packets.

9. The next LMP sends an ACK message to the original LMP.

10. The original LMP starts to forward all of the unsent packets.

11. The next LMP sends the forwarded packets to the $\mathrm{MMH}$.

12. The next LMP sends the message to the original LMP and terminates the connection between the original LMP and itself.

13. The next LMP sends the packets received from the MS to the MMH. After the proxy handoff procedure is done, the system goes into the normal transmission procedure.

\section{PERFORMANCE ANALYSIS}

Proxy Handoff is proposed to generalize the process of switching from one LMP to another LMP. The first experiment is to verify whether the proposed $\mathrm{PH}$ is required and effective or not. Fig. 1 and Fig. 2 depict the experimental results without APH and with APH, respectively. The experiment without APH means that MMHs do not perform PH. In both figures, the y-axis denotes the number of packets that are received in one time unit, which is one second in this experiment; the $\mathrm{x}$-axis denotes the kth time unit.

In each time unit, the Diamond shape data points denotes those packets that are received and on time the Circle shape data points denotes those packets that are lost and not on time. If packets are lost, it implies that the quality of presentation should be affected drastically. This packet lost is show in graph.

TRAFFIC WITH $250 \mathrm{~kb}$

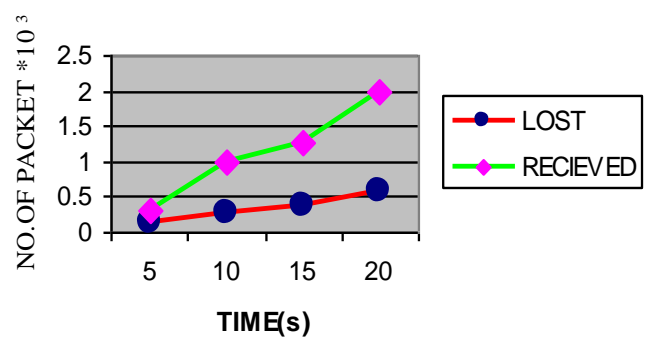

Fig .1(a) 
TRAFFIC WITH $500 \mathrm{~Kb}$

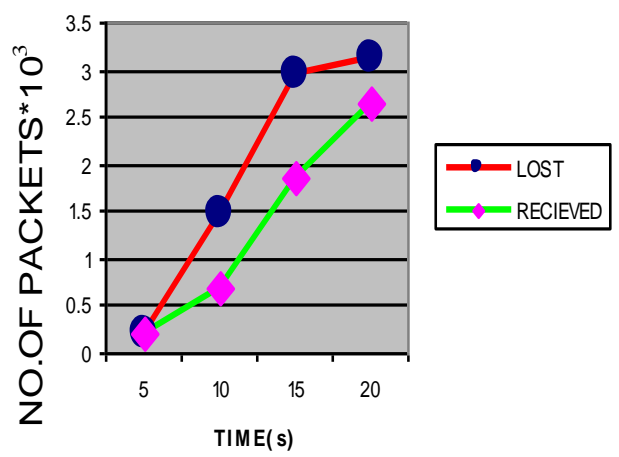

Fig.1 (b)

TRAFFIC WITH $750 \mathrm{~Kb}$

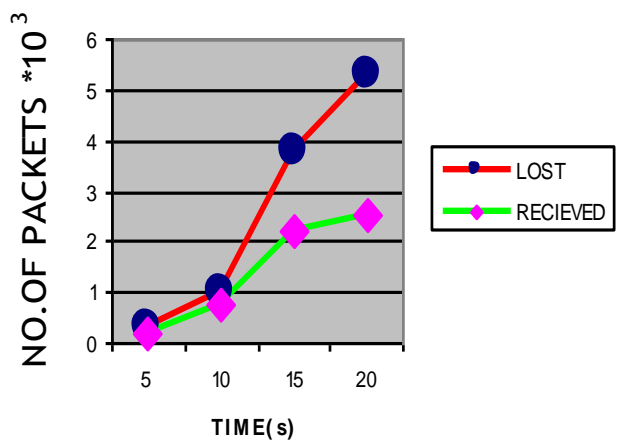

Fig.1(c)

TRAFFIC WITH $1000 \mathrm{~Kb}$

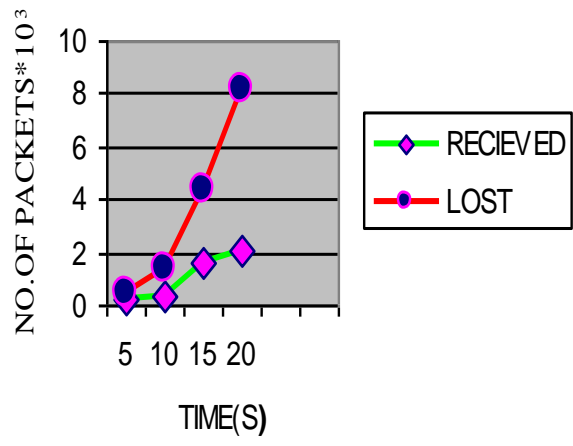

Fig. 1(d)

Fig.1. The transmission quality without PH. (a) With $250 \mathrm{~kb}$ background traffic. (b) With $500 \mathrm{~kb} / \mathrm{s}$ background traffic. (c) With $750 \mathrm{~kb} / \mathrm{s}$ background traffic. (d) With $1000 \mathrm{~kb} / \mathrm{s}$ background traffic.

In Fig. 1, the transmission quality without PH is worse and worse as the background traffic becomes heavier and heavier.
When the background traffic is not more than $250 \mathrm{~kb} / \mathrm{s}$ (Figs. 1a), very less packet loss happens and only a few packets are received out of order. When the Background traffic is $500 \mathrm{~kb} / \mathrm{s}$ (Fig.1b), packet loss rate is increased slowly and received packets are out of order. When the background traffic is 750 $\mathrm{kb} / \mathrm{s}$ (Fig. 1c), packets are lost and almost all received packets are out of order. When the background traffic is $1000 \mathrm{~kb} / \mathrm{s}$ (Fig. 1d), received packets are totally reduced and loss rate is increased drastically. This implies that the quality of service is affected drastically.

\section{TRAFFIC WITH $250 \mathrm{~Kb}$}

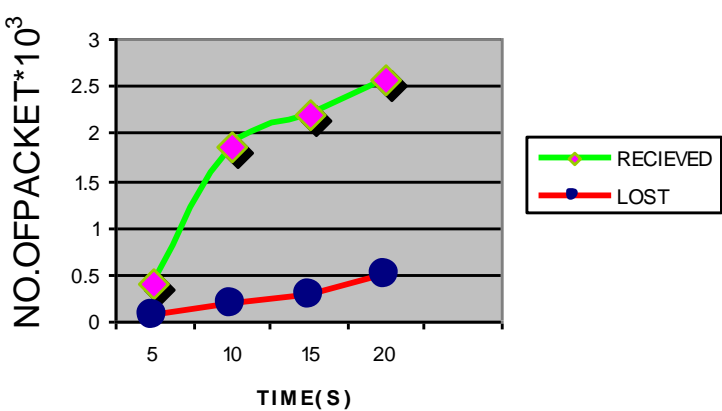

Fig .2(a)

TRAFFIC WITH $750 \mathrm{~Kb}$

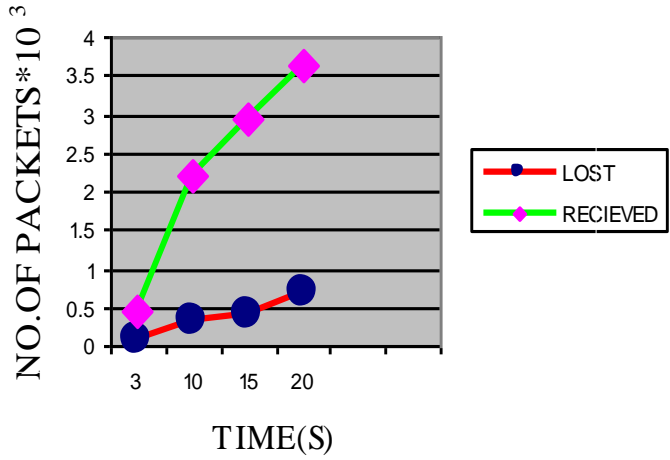

Fig .2(b)

TRAFFIC WITH 1000

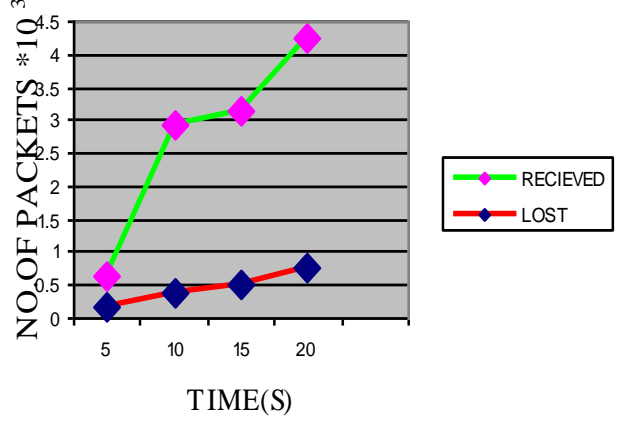

Fig .2(c) 
Fig. 2 The transmission quality with PH. (a) Without background traffic. (b) With $2 \mathrm{Mb} / \mathrm{s}$ background traffic. (c)With $4 \mathrm{Mb} / \mathrm{s}$ background traffic. (d) With $6 \mathrm{Mb} / \mathrm{s}$ background traffic.

In each time unit, the Diamond shape data points denotes those packets that are received and on time the Circle shape data points denotes those packets that are lost and not on time. In Fig. 2, the transmission quality keeps steady even if the background traffic becomes heavier and heavier. For example, when the background traffic is $750 \mathrm{~kb} / \mathrm{s}$ (Fig.2c), percentage of received packets are high and percentages of lost packets are low and also packets are in order. When the background traffic is $1000 \mathrm{~kb} / \mathrm{s}$ (Fig. 2d), 7.43 percent packets are lost and 5.99 percent packets are out of order. Moreover, most packets are lost in the initial period, i.e.,the period of the APH process. After the APH process, the transmission quality remains steady. From this experiment, we can conclude that $\mathrm{APH}$ is effective to keep the transmission quality of MMHs steady when MMHs are moving.

\section{CONCLUSION}

In the evolution of mobile networks beyond 3G, multimedia streaming is becoming more and more urgent. When the three-tier system architecture is applied to mobile networks for improving the transmission quality, it must take user mobility into consideration. In this paper, we have proposed a new mechanism called Application-layer Proxy Handoff (APH). The goal of APH is to let applications perform L7 handoff smoothly when a client keeps moving under the three-tier architecture. Our contributions are as follows:

- To generalize the vital issues of switching to a new proxy which are 1) how to select the next proxy and 2) how to recover the session on the next proxy. Unlike the past works, we distill the switching process to be independent of L2 and/or L3 handoff.

- We introduce the procedure of switching to a new proxy and provide the complete solution in APH using 1) application-layer anycast and 2) IPv6 multicast. Unlike the past works, they may focus on only one of the two vital issues.

- The corresponding algorithms, procedures, and Performance of APH have been described step by step in detail.

\section{ACKNOWLEDGMENT}

We would like to thank all the faculty of the department for their unstinting support .Thanks also to the anonymous reviewers who provided many useful comments for the improvement of our paper.

\section{REFERENCES}

[1] Chung-Ming Huang, Member, IEEE, and Chao- Hsien Lee, "Layer 7 Multimedia Proxy Handoff Using Anycast/Multicast in Mobile Networks," IEEE Trans.ON on mobile computing, vol. 6, no. 4, APRIL 2007, pages: 411-422.
[2] Prof. S. Nandakumar, Rahul Singh, Sanjeet Singh, "Traffic Driven \& Received Signal Strength Adaptive

Handoff Scheme," International Journal of Computer Applications (0975 - 8887) Volume 21- No.6 May 2011.

[3] Prof. S. K. Radhika, Dr. A. Venugopal Reddy, "Vertical Handoff Decision using Game Theory Approach for Multi-mode Mobile Terminals in Next Generation Wireless Networks," International Journal of Computer Applications (0975 - 8887) Volume 36- No.11 December 2011.

[4] N.Montavont and T. Noel, "Handover Management for Mobile Nodes in IPv6 Networks," IEEE Comm. Magazine, vol. 40, no. 8, pp. 38-43, Aug. 2002.

[5] J. Tourrilhes, "L7-Mobility: A Framework for Handling Mobility at the Application Level," Proc. 15th IEEE Int'1 Symp. Personal, Indoor and Mobile Radio Comm. (PIMR'04), vol. 2, pages. 1246-1251, 2004.Networks,' IEEE Comm. Magazine, vol. 40, no. 8, pp. 38-43, Aug. 2002

[6] H.Schulzrinne and E. Wedlund, "Application-Layer Mobility Using SIP," ACM SIGMOBILE Mobile Computing and Comm. Rev.vol. 4, no. 3, pages. 47-57, 2000

[7] C. Lin, H. Lee, C. Chan, and J. Wang, "Cooperative Proxy Framework for Layered Video Streaming," Proc. GLOBECOM,vol. 1, pages. 251-255, 2005.

[8] K. Kim, H. Lee, and K. Chung, "A Distributed Proxy Server System for Wireless Mobile Web Service," Proc. 15th IEEE Int'l Conf. Information Networking, pages. 749-754, Feb. 2001.

[9] W.Gu and A.S. Helal, "Extended Internet Caching Protocol-A Foundation for Building Ubiquitous Web Caching," Proc. ACM Symp. Applied Computing (SAC '03), pages. 901-907, Mar. 2003.

[10] A. Dutta, J. Chennikara, W. Chen, O. Altintas, and H. Schulzrinne,"Multicasting Streaming Media to Mobile Users," IEEE Comm. Magazine, vol. 41, no. 10, pp. 8189, Oct. 2003.

[11] Z.Fei, S. Bhattacharjee, E.W. Zegura, and M.H. Ammar, "A Novel Server Selection Technique for Improving the Response Time of a Replicated Service," Proc. INFOCOM, vol. 2, pp. 783-791, 1998.

[12] E.W. Zegura, M.H. Ammar, Z. Fei, and S. Bhattacharjee, Application-Layer Anycasting: A Server Selection Architecture and Use in a Replicated Web Service," IEEE/ACM Trans. Networking, vol. 8, no. 4, pp. 455466, 2000.

[13] K.McKinley, Udiyan I. Padmanabha, Nandagopal Ancha, and Seyed Masoud Sadjadi, "Composable Proxy Services to Support Collaboration on the Mobile Internet," IEEE TRANSACTIONS ON COMPUTERS, VOL. 52, NO. 6, JUNE 2003.

[14] Vincenzo Mancuso, Giuseppe Bianchi, "Streaming for Vehicular Users via Elastic Proxy Buffer Management" IEEE Communications Magazine • November 2004. 